\title{
BMJ Open Maximising trichiasis surgery success (MTSS) trial: rationale and design of a randomised controlled trial to improve trachomatous trichiasis surgical outcomes
}

\author{
Belay Bayissasse, ${ }^{1}$ Kristin M Sullivan, ${ }^{2}$ Shannath L Merbs, ${ }^{3,4}$ Beatriz Munoz, ${ }^{4}$ \\ Alexander Keil, ${ }^{2,5}$ Alemayehu Sisay, ${ }^{1}$ Alison Singer, ${ }^{2}$ Emily W Gower (D) ${ }^{2,6}$
}

To cite: Bayissasse B, Sullivan KM, Merbs SL, et al. Maximising trichiasis surgery success (MTSS) trial: rationale and design of a randomised controlled trial to improve trachomatous trichiasis surgical outcomes. BMJ Open 2020;10:e036327. doi:10.1136/ bmjopen-2019-036327

- Prepublication history and additional material for this paper are available online. To view these files, please visit the journal online (http://dx.doi org/10.1136/bmjopen-2019036327).

Received 13 December 2019 Revised 31 January 2020 Accepted 17 February 2020

Check for updates

(C) Author(s) (or their employer(s)) 2020. Re-use permitted under CC BY-NC. No commercial re-use. See rights and permissions. Published by BMJ.

For numbered affiliations see end of article.

Correspondence to

Dr Emily W Gower;

egower@unc.edu

\section{ABSTRACT}

Introduction Trachomatous trichiasis (TT) is a condition in which the eyelid turns inward and eyelashes abrade the front part of the eye. To prevent eventual blindness, surgery is recommended. Two surgical procedures are commonly used, bilamellar tarsal rotation (BLTR) and posterior lamellar tarsal rotation (PLTR). Evidence suggests that incision height and surgery type may affect the risk of postoperative $\Pi$ (PTT) and other surgical outcomes. However, these studies have not prospectively compared the impact of incision height on surgical outcomes.

Methods and analysis Maximising trichiasis surgery Success (MTSS) is a three-arm, randomised clinical trial being conducted in Ethiopia. Participants will be randomly assigned on a 1:1:1 basis to BLTR with a $3 \mathrm{~mm}$ incision height, BLTR with a $5 \mathrm{~mm}$ incision height, or PLTR $3 \mathrm{~mm}$ incision height. Patients are eligible for the trial if they have previously unoperated upper eyelid TT. Follow-up visits will be conducted by trained eye examiners at 1 day, 2 weeks, 6 weeks and 12 months after surgery. The primary outcome is incident PTT within 1 year following surgery. Logistic regression will be used in an intention-to-treat analysis to assess outcome incidence by surgical approach.

Ethics and dissemination The University of North Carolina and Johns Hopkins School of Medicine institution review boards, Ethiopian National Research Ethics Review Committee and Ethiopian Food, Medicine, Healthcare and Administration and Control Authority provided ethics approval for the trial. On completion, trial results will be disseminated at local and international meetings and in peer-reviewed journals.

Trial registration number NCT03100747.

\section{INTRODUCTION}

Trachoma is the leading infectious cause of blindness worldwide, with an estimated 1.2 million people irreversibly blind and 2 million people visually impaired. ${ }^{1}{ }^{2}$ Repeated infections with the bacterium Chlamydia trachomatis, primarily during childhood, result in chronic inflammation of the eyelids. In some cases, this inflammatory process leads to tarsal
Strengths and limitations of this study

- The Maximising Trichiasis Surgery Success Trial is the first randomised clinical trial to assess the importance of incision height in trichiasis surgery.

- Due to the nature of the treatment groups, surgeons will not be blinded to treatment assignment.

- The extensive use of photography allows for close monitoring of treatment assignment and outcomes assessments.

- The use of both newly trained surgeons and those who were first trained in bilamellar tarsal rotation and later in posterior lamellar tarsal rotation allows for assessment of the importance of a learning curve and prior experience in surgical outcomes.

conjunctival scarring which can cause the eyelid to rotate inward causing inturned eyelashes to abrade the front part of the eye, a condition known as trachomatous trichiasis (TT). If left untreated, TT leads to corneal opacity and subsequent vision loss. An estimated 2.5 million people are in need of TT management in order to prevent irreversible vision loss. ${ }^{3}$

Two surgical procedures are commonly used to correct TT: the bilamellar tarsal rotation procedure (BLTR) and the posterior lamellar tarsal rotation procedure (PLTR). ${ }^{4}$ Each procedure includes either a partial or full-thickness incision through the eyelid, which then allows the TT to be corrected by rotating the trichiatic lashes back to their normal position. Because of the large caseload and the limited number of ophthalmologists in trachoma-endemic countries, surgery is typically performed by nurses after receiving a $2-4$ week training on TT surgical techniques.

While surgery is often effective at relieving $\mathrm{TT}$, in some instances, the surgery is not 
correctly performed, and the patient has eyelashes touching the eye at an early postoperative visit. In other instances, the disease progresses, and eyelashes again become inturned over time. In both of these cases, eyelashes touching the eye are referred to as postoperative trichiasis (PTT). While some trials have reported PTT incidence as low as $10 \% 1$ year after surgery, ${ }^{5}$ in other settings it was estimated to reach as high as $60 \%$ within a few years after surgery. ${ }^{4-14}$ PTT-affected eyes need further surgical correction. Other poor surgical outcomes include granuloma formation and the development of eyelid contour abnormalities. ${ }^{6}{ }^{15-19}$ As countries work to eliminate their TT surgical backlog, high rates of surgical participation are necessary, and this is more likely to be achieved if early surgical outcomes are good. Hence, identifying ways to ensure high-quality TT-surgery outcomes remains an important public health issue.

The Maximising Trichiasis Surgery Success (MTSS) trial was designed to evaluate first-time TT surgical outcomes by comparing three TT surgical procedures: PLTR, BLTR following the current WHO guideline of a $3 \mathrm{~mm}$ incision height and BLTR using a $5 \mathrm{~mm}$ incision height. Here, we describe the rationale and methods used to conduct this randomised clinical trial of nearly $5000 \mathrm{TT}$ participants.

\section{Rationale}

Until recently, the WHO recommended that national blindness prevention programme train new TT surgeons to perform the BLTR procedure. ${ }^{20}$ In the Partnership for Rapid Elimination of Trachoma (PRET)-surgery randomised trial of BLTR surgeries comparing surgery using hemostats versus surgery with the TT clamp, ${ }^{21}$ PTT risks at 24 months ranged from $36.6 \%-43.2 \%{ }^{6}$ In investigating reasons for the frequent occurrence of PTT in that trial, our team collected evidence that suggested the recommended $3 \mathrm{~mm}$ incision height may not be optimal. We found that eyelids with a higher incision scar height 1 year after surgery had a lower PTT incidence than eyelids with a lower incision scar height. ${ }^{22}$ Thus, we proposed a randomised clinical trial to compare $5 \mathrm{~mm}$ target incision heights versus $3 \mathrm{~mm}$ target incision heights, both with the BLTR procedure.

During the trial planning period, new clinical trial results became available, reporting that at 1 year after surgery PLTR recipients had nearly a $50 \%$ lower occurrence rate of PTT than BLTR recipients (22\% vs 13\%; adjusted OR 1.96; 95\% CI: 1.40 to 2.75$).{ }^{9}$ However, within that trial, 1-year incision scar measurement data were consistent with the PRET-surgery results, indicating that higher BLTR incision scars were associated with a lower PTT incidence. That trial used surgeons who were trained in PLTR and later received conversion training to perform BLTR. Following the release of the PLTR versus BLTR trial results, two meetings of the global TT community were held. At both meetings, experts strongly recommended that another trial should be conducted using BLTR surgeons who receive conversion training to PLTR, and a PLTR arm was added to the trial described here.

\section{METHODS AND ANALYSIS}

\section{Study overview}

This study is a three-arm randomised, superiority trial of approximately 6900 eyes of participants presenting for first-time TT surgery.

Participants will be randomised on a 1:1:1 basis to BLTR surgery with a $3 \mathrm{~mm}$ incision height, BLTR surgery with a $5 \mathrm{~mm}$ incision height, or PLTR surgery. When participants present with bilateral TT they will receive the same procedure on both eyes. Follow-up will be conducted at 1 day, 2 weeks, 6 weeks and 12 months postsurgery. At each follow-up visit, participants will be assessed for evidence of the following outcomes: PTT, granuloma formation and eyelid contour abnormalities. ${ }^{23}$ All outcomes will be assessed by a trained grader who is blinded to intervention assignment.

\section{Specific aims and outcomes}

The specific aims of this trial are to examine whether the 1 year risks of PTT, eyelid contour abnormalities and granulomas differ significantly across surgical approaches to TT correction. For each aim, we will compare (1) BLTR surgeries using a $3 \mathrm{~mm}$ incision height versus BLTR incisions using a $5 \mathrm{~mm}$ incision height, (2) PLTR surgery versus BLTR with a $3 \mathrm{~mm}$ incision height and (3) PLTR surgery versus BLTR with a $5 \mathrm{~mm}$ incision height.

The primary outcome is incident PTT within 1 year after surgery. Secondary outcomes include incident eyelid contour abnormalities and incident pyogenic granuloma formation within 1 year after surgery. We hypothesise that increasing the BLTR incision height from $3 \mathrm{~mm}$ to $5 \mathrm{~mm}$ will reduce the risk of PTT. Further, we hypothesise that the risk of PTT will not be significantly different when comparing BLTR with a $5 \mathrm{~mm}$ incision height versus PLTR. Finally, we hypothesise that the risk of granulomas and eyelid contour abnormalities will be similar across the groups.

\section{Setting}

This study will take place in the Hadiya, Gedio and Keffa zones and the Yem, Amaro and Burji woredas of Southern Nations Nationalities and Peoples Regional State of Ethiopia. We selected Ethiopia for this study because of its large TT backlog and presence of functional TT surgery programme. $^{3}$

\section{Recruitment and eligibility}

We will identify potential participants through two sources, community-based TT case finders and community announcements. Community-based TT case finders will receive a half-day training on how to identify TT cases and will be paid a small fee for their services. These case finders will go house-to-house within their neighbourhood and examine everyone in the household for presence of TT. Identified patients will be asked to present at the community screening site on the day of the surgical camp. TT patients will also be identified through community announcements. Village criers will use a megaphone at marketplaces and 
spiritual gatherings to announce the date and time for a community screening.

On the day of the local surgical camp, a TT surgeon will go to each community screening site and screen all suspected TT cases. Typically, patients needing TT surgery will be transported to the surgery site using a study vehicle. At the surgical camp, the study staff screen all suspected TT cases for the presence of operable TT. To clearly identify study-eligible eyelids, the screener will use a black marker to mark each hand of the case with 'TT,' 'PTT,' or 'X' (neither TT nor PTT) to indicate the diagnosis for each eye.

A study team member will meet with each potential participant to determine eligibility, provide information about the study and ask if they are interested in participating. To be eligible, a participant must meet all the following criteria.

1. At least one upper eyelid with the presence of previously unoperated TT (defined by at least one eyelash touching the globe or evidence of epilation).

2. Aged 18 or older (in order to provide consent as an adult).

3. Willingness to comply with all study procedures for the duration of the study.

Interested individuals will meet with a consent specialist who will review the study procedures and read the consent form (online supplementary file) in the local language. Individuals who agree to participate will provide either their signature or a thumbprint. Table 1 highlights key activities at this and subsequent study visits.

\section{Randomisation and blinding}

Randomisation assignments will be predetermined for each participant identification number and will be concealed until the time of surgery. First, a custom randomisation programme will be developed to assign participants on a 1:1:1 basis to BLTR surgery with a target $3 \mathrm{~mm}$ incision height, BLTR surgery with a target $5 \mathrm{~mm}$ incision height, or PLTR, using permuted block sizes of 6 and 12. Then, the study arm assignment and corresponding participant identification number will be preprinted on a surgical evaluation form and placed in an opaque, letter-sized envelope with the corresponding participant identification number on the outside. These randomisation envelopes will be prepared and sealed at the University of North Carolina (UNC).

In Ethiopia, other study forms will be prepared and placed into larger envelopes labelled with participant identification numbers on the outside. When the randomisation envelopes are received in Ethiopia, they will be placed in the corresponding larger participant envelopes. The randomisation envelopes will be opened by the surgical assistant when the participants are in the operating room.

The participants and the outcomes assessment team will be blinded to treatment assignment. In order to ensure the outcome examiners remain blinded, they will not present at the surgical site and will not have access to data from the baseline and surgical visit when conducting follow-up assessments.
Table 1 Timeline of major study procedures

\begin{tabular}{|c|c|c|}
\hline Time point & Activity & Description of activities \\
\hline Visit 1: Day 0 & $\begin{array}{l}\text { Baseline exam } \\
\text { and surgery }\end{array}$ & $\begin{array}{l}\text { Eligibility review } \\
\text { Written informed consent } \\
\text { Baseline ocular exam } \\
\text { (severity of T,, visual } \\
\text { acuity, photographs) } \\
\text { Study intervention: } \\
\text { randomisation and } \\
\text { surgery } \\
\text { Intraoperative data } \\
\text { collection (surgical } \\
\text { procedure used, incision } \\
\text { height measurement, } \\
\text { photographs and any } \\
\text { complications) }\end{array}$ \\
\hline $\begin{array}{l}\text { Visit 2: } \\
\text { 1-day post-op } \\
\text { exam }\end{array}$ & $\begin{array}{l}\text { Patch removal } \\
\text { and correction } \\
\text { surgery }\end{array}$ & $\begin{array}{l}\text { Bandage removal } \\
\text { Examination of eyes for } \\
\text { any problems } \\
\text { Surgical correction if } \\
\text { needed (for severe under } \\
\text { or over-correction) } \\
\text { Photograph of eyelid }\end{array}$ \\
\hline $\begin{array}{l}\text { Visit 3: } \\
\text { 2-week post-op } \\
\text { exam }\end{array}$ & Suture removal & $\begin{array}{l}\text { Sutures removed } \\
\text { Ocular exam to evaluate } \\
\text { the presence of TT, eyelid } \\
\text { contour abnormalities } \\
\text { and granuloma formation } \\
\text { Photographs of eyelid }\end{array}$ \\
\hline $\begin{array}{l}\text { Visit 4: } \\
\text { 6-week post-op } \\
\text { exam }\end{array}$ & Ocular exam & $\begin{array}{l}\text { Ocular exam to evaluate } \\
\text { the presence of TT, eyelid } \\
\text { contour abnormalities } \\
\text { and granuloma formation } \\
\text { Photographs of eyelid }\end{array}$ \\
\hline $\begin{array}{l}\text { Visit 5: } \\
\text { 12-month post- } \\
\text { op exam }\end{array}$ & Ocular exam & $\begin{array}{l}\text { Final assessment of } \\
\text { participants: } \\
\text { Ocular exam to evaluate } \\
\text { the presence of TT, eyelid } \\
\text { contour abnormalities } \\
\text { and granuloma formation } \\
\text { Visual acuity assessment } \\
\text { Photographs of eyelid }\end{array}$ \\
\hline
\end{tabular}

post-op, postoperative; $\Pi$, trachomatous trichiasis.

\section{Intervention}

\section{Training of TT surgeons}

We will employ two groups of surgeons: (i) those newly trained simultaneously on BLTR and PLTR procedures and (ii) those originally trained to perform BLTR surgery but later trained on PLTR surgery. This latter group, conversion surgeons, is required to perform at least 100 PLTR surgeries prior to performing MTSS surgeries. At the start of the trial, all surgeons in the study area will be assessed by an external examiner, using the WHO's checklist for final assessment of TT surgeons. ${ }^{4}$

All surgeons will receive additional training on incision height marking and measurement. Training will begin with the use of a surgical simulation device, the Human Eyelid Analogue Device for Surgical Training and Skill Reinforcement in Trichiasis Surgery (HEAD START), ${ }^{24}{ }^{25}$ to 
practice measuring and marking the incision height. Once surgeons are skilled at marking and measuring the heights using HEAD START, they will mark and measure heights on patients who will receive surgery. A kappa of $>0.7$ for measuring the incision height on patients will be required before the surgeon is able to perform study-related surgeries.

\section{TT surgery}

All participants will receive TT surgery with either the BLTR or PLTR procedure, as outlined in the WHO's Trichiasis surgery for trachoma manual. ${ }^{4}$ The BLTR $5 \mathrm{~mm}$ incision group surgeries will be performed as described in the manual, but with the incision placed $5 \mathrm{~mm}$ above the eyelid margin. BLTR surgeries will be performed using the largest TT clamp possible. ${ }^{21}$ If the smallest clamp will not fit, the surgeon will perform the PLTR procedure instead.

\section{Quality assurance during surgery}

The surgeon will measure and mark the target incision height for BLTR surgeries as follows. After applying the TT clamp, the surgeon will place a calliper with one point at the eyelash line and the other 3 or $5 \mathrm{~mm}$ above the eyelash line and will mark three dots (nasally, centrally and temporally) at the appropriate height using a sterile pen. The dots will be connected with a series of additional dots to mark the target incision across the width of the eyelid. The surgical assistant will then take a picture of the marking while the surgeon held a ruler against the eyelid.

For all three groups, the surgeon will measure the incision height from the cut edge of the tarsus to the eyelash line nasally, centrally and temporally after completing the incision. For the BLTR procedure, the TT clamp will be removed and used to aid in everting the eyelid for the measurement. For PLTR surgeries, the PLTR plate will remain in place during the measurement. The surgical assistant will photograph each measurement.

Throughout recruitment, we will regularly monitor incision height reporting by reviewing a $10 \%$ sample of central incision measurements for each surgeon in a blinded fashion. A grader will examine each photograph and report the incision height based on the ruler placement. If the ruler is not placed appropriately, the grader will report an estimated incision height, based on their judgement of how far the ruler is from the cut edge of the incision. These measurements will be compared against the surgeon-reported incision height. When more than $10 \%$ of the measurements have a discrepancy of $>0.5 \mathrm{~mm}$, the field team will perform retraining to ensure that incision markings will be correctly placed and that the true incision height will be reported.

\section{Adverse event monitoring}

At the end of each surgery, the surgeon will complete a surgical evaluation form on which he will indicate if any of the listed common adverse events have occurred. In addition, at each follow-up visit, the team will collect information on adverse events, including any hospitalisations and deaths. If any are identified, an adverse event form will be completed. Given the nature of the one-time treatment for this study and the anticipated rapid rate of recruitment, the Data Safety Monitoring Committee determined that no stopping rules will be necessary.

\section{Data collection and outcome assessments Preoperative data collection}

Preoperatively, we will measure distance visual acuity using Peek Acuity software (https://www.peekvision.org/) on a smartphone, at $4 \mathrm{~m}$, moving to $2 \mathrm{~m}$ as needed. The examiner then will assess the TT status of each upper and lower eyelid using 2.5 X binocular loupes and a flashlight. Data collected will include the number and location of trichiatic eyelashes (nasal, central and temporal) and evidence of epilation for each eyelid. Corneal scarring will be assessed using a standard detailed grading scheme. ${ }^{26}$ Conjunctivalisation of the eyelid margin will be assessed using previously developed procedures. ${ }^{27}$ All data will be collected electronically using customised Open Data Kit 9 (https://opendatakit.org/) forms on Motorola Moto X Pure Edition or Samsung Galaxy S8 smartphones. These phones will also be used to take photographs of the eyelids, cornea and tarsal conjunctiva.

\section{Follow-up data collection}

An outcomes examiner will evaluate participants 1 day, 2 weeks, 6 weeks and 12 months postoperatively (table 1). At the 1-day visit, the examiner will remove the patches and clean and examine the operated eyelids. Severe overcorrection, where the cut edge of the tarsus is visible or severe under-correction, where eyelashes are still touching the globe, will be addressed with additional corrective surgery. At the 2 week visit, the examiner will remove the sutures.

At the 2 week, 6 week and 12 months visits, the examiner will assess each eyelid for the presence of study outcomes (PTT, granuloma and eyelid contour abnormalities) and take pictures of the upper eyelids with the eyelid margins visible. If PTT is present, the examiner will document the number and location of trichiatic eyelashes as well as the extent of epilation and its location(s): nasal, central and temporal. If granuloma is present, the examiner will record if it is removed and if suture material is present. The examiner will grade eyelid contour abnormalities as mild, moderate or severe, using the classification previously developed. ${ }^{23}$ At the 12 month visit, the examiner will record the incision scar height.

Participant retention will be maintained through two means. First, we will provide each participant with a reminder card at each visit. These cards will include the date and location of the next follow-up visit. In addition, about 2 weeks before an upcoming visit, the outcome examiner will call the local community health worker who lives in the participant's village. The examiner will remind the health worker of the date and location of the participant's next exam. 
A coordinating centre-based photograph grader will review photographs of all eyelids to assess presence of eyelid contour abnormality.

\section{Data management}

All data will be collected by certified staff members who have completed human subjects' research training and a multi-day training course related to the trial procedures. Data will be managed using a Microsoft Access database designed specifically for this trial. Paper-based forms will be entered directly into the database. Data will be collected electronically using smartphones and uploaded to the database daily via USB tethering. The database will provide reports to monitor recruitment and follow-up status and data consistencies for ongoing immediate monitoring. The data will be sent regularly to UNC using a secure file transfer protocol where they will be further evaluated for accuracy and completeness.

\section{Data and Safety Monitoring Committee}

The Data and Safety Monitoring Committee (DSMC) includes four voting members and a non-voting member from the funding organisation, the US National Eye Institute. The DSMC is independent from the sponsor and has no competing interests. The committee consists of experts in ophthalmology, biostatistics and clinical trials conduct, as well as an expert on the local context. The DSMC will meet at least twice annually, either in-person or through teleconference. While there are no formal stopping guidelines, the DSMC will review blinded study data annually. If deemed necessary, they will review the data in a nonblinded fashion.

In addition to the DSMC, the Ethiopian ethics committee will monitor the trial at least once during follow-up.

\section{Patient and public involvement}

Due to the technical nature of the study design, trichiasis surgery patients will not be involved in the development of the methods for this trial. However, we will seek involvement from local ophthalmologists as well as other public health practitioners in Ethiopia and abroad.

\section{Statistical analysis plan \\ Sample size}

We designed this study to have $80 \%$ power to detect a $25 \%$ relative risk difference for each of the pairwise primary outcomes, dividing the two-sided alpha of 0.05 between the three comparisons (0.0167 each). Assuming a PTT incidence of $15 \%$ in the BLTR $3 \mathrm{~mm}$ group and $5 \%$ loss to follow-up, the trial required a sample size of 6920 eyelids. Given that two eyes of a participant could be enrolled, we used an intraclass correlation of 0.24 based on the correlation between two eyes of a participant in our prior studies. Assuming $40 \%$ would be bilateral, an estimated 5001 participants would be needed to meet our eyelid sample size target.
Primary analyses

The primary analyses will comprise three pairwise comparisons of cumulative PTT incidence, defined as any occurrence of PTT noted at 6 weeks or 12 months. To account for the clustering of outcomes across individuals, we will perform pairwise comparisons using a logistic mixed model that includes normally-distributed random effects for participant and fixed effects for treatment arms. A similar model will be developed for eyelid contour abnormalities based on the presence of eyelid contour abnormalities at the 1-year follow-up visit. Cumulative incidence of granuloma, defined as the occurrence of granuloma at any postsurgical study visit, will also be analysed using such a model. For each of these primary analyses, statistical significance for the tests comparing differences between study arms will be determined based on an alpha level of 0.0167 to account for the multiple testing that results from using pairwise comparisons. We will assume that loss-tofollow-up $(5 \%)$ has negligible impact on the OR and will perform analyses among study participants with complete outcomes data.

\section{Secondary analyses}

\section{As-treated analysis of primary outcomes}

To allow that some surgical procedures may not comport with assigned study arm, we will use a logistic mixed model (again with nested random effects) to perform an as-treated analysis contrasting the cumulative incidence odds of PTT across the three surgical approaches evaluated in this study (rather than the study arm). To allow that actual incision heights may vary within incision height assignments, we will perform an additional as-treated analysis limited to BLTR participants in which we compare lower incisions with higher incisions, where the cut-point between low and high will be determined empirically, or the analysis will be done allowing for continuous incision height (encoded flexibly using a spline) if no logical cut-point can be identified.

\section{Modifiers of effectiveness}

We will assess the impact of treatment-related modifiers of treatment effectiveness using logistic mixed models of surgical outcomes. We will use these models to perform within-study-arm analyses contrasting the cumulative incidence of PTT and the prevalence of eyelid contour abnormalities at 1 year among those with surgical corrections at the 1-day visit with the same measures among those without corrections.

Additionally, we will compare cumulative PTT incidence among BLTR participants across different scar height measurements at the 1-year follow-up visit. This model additionally will adjust for baseline incision height so that it will implicitly assess whether eyelid remodelling that results in changes in scar height is associated with PTT. We will also address associations between scar height changes and study outcomes by modelling the difference between scar height at 1 year post surgery and reported incision height as a function of the presence of PTT or eyelid contour abnormalities at the 1-year follow-up visit. 
Table 2 Trichiasis severity classification

\section{TT severity} classification

$\begin{array}{ll}\text { Mild } & 1-4 \text { eyelashes touching globe, no epilation } \\ & \text { OR } \\ & <1 / 3 \text { of eyelid epilated, no eyelashes } \\ & \text { touching globe } \\ \text { Moderate } & 5-9 \text { eyelashes touching globe, no epilation } \\ & \text { OR } \\ & 1-4 \text { eyelashes touching globe and }<1 / 3 \text { of } \\ & \text { eyelid epilated } \\ & 5-9 \text { eyelashes touching globe and any } \\ \text { Severe } & \text { OR } \\ & \text { OR } \\ & \text { epilation } \\ & \text { OR } \\ \geq 1 / 3 \text { of eyelion status }\end{array}$

Source: Gower et al ${ }^{6}$

$\Pi$, trachomatous trichiasis.

Finally, we will assess whether surgical outcomes differ by surgeon training by contrasting outcomes among participants treated by surgeons trained originally in BLTR with outcomes among participants treated by surgeons who learnt BLTR and PLTR simultaneously, which we will assess by including product terms between training ( $1=$ trained in BLTR first, $0=$ trained in both simultaneously) and study arm in our primary models.

\section{PTT outcome severity}

Using a similar modelling approach to our other analyses, we will assess whether PTT severity (defined in table 2) or location differs by treatment arm among those with PTT at the 1-year follow-up visit.

\section{Exploratory and descriptive analyses}

We will perform several additional analyses to explore the characteristics of participants and study eyelids and to generate hypotheses for future studies. Key analyses are described here. Request for details on additional planned analyses can be sent to the study team. First, we will assess changes in eyelid contour abnormalities by first describing the extent and direction of changes in eyelid contour abnormalities over time, and then performing an exploratory analysis of the characteristics of eyelids with changes over time by modelling the association between the change in contour abnormalities (yes/no) as a function of these characteristics. Second, we will explore methods for distinguishing how much of the effect of surgical outcome could be moderated by correlation of outcomes within individuals, as well as methods for quantifying the inter-individual correlation in study outcomes among participants with bilateral surgeries. Third, we will use photograph grading to assess the consistency and repeatability of our entropion grading system by having
Table 3 Trial registration data and protocol summary

\begin{tabular}{|c|c|}
\hline Data category & Information \\
\hline $\begin{array}{l}\text { Primary registry and trial } \\
\text { identifying number }\end{array}$ & $\begin{array}{l}\text { ClinicalTrials.gov Identifier: } \\
\text { NCT03100747 }\end{array}$ \\
\hline First submitted date & Feb 27, 2017 \\
\hline $\begin{array}{l}\text { Secondary identifying } \\
\text { numbers }\end{array}$ & $\begin{array}{l}\text { 16-2878 } \\
\text { UG1EY025992 (US NIH Grant/ } \\
\text { Contract) }\end{array}$ \\
\hline $\begin{array}{l}\text { Sources of monetary } \\
\text { support }\end{array}$ & $\begin{array}{l}\text { National Eye Institute-National } \\
\text { Institutes of Health }\end{array}$ \\
\hline Primary sponsor & $\begin{array}{l}\text { National Eye Institute-National } \\
\text { Institutes of Health }\end{array}$ \\
\hline Contact for queries & $\begin{array}{l}\text { Emily Gower, PhD, (egower@ } \\
\text { unc.edu) }\end{array}$ \\
\hline Title & $\begin{array}{l}\text { Maximising TT surgery success } \\
\text { trial }\end{array}$ \\
\hline Countries of recruitment & Ethiopia \\
\hline $\begin{array}{l}\text { Health condition(s) or } \\
\text { problem(s) studied }\end{array}$ & Trachomatous trichiasis (TT) \\
\hline Interventions & $\begin{array}{l}\text { Group 1: Bilamellar tarsal } \\
\text { rotation procedure (BLTR) } \\
\text { with incision } 3 \mathrm{~mm} \text { from the } \\
\text { eyelid margin (standard BLTR } \\
\text { procedure) } \\
\text { Group 2: BLTR with incision } 5 \\
\text { mm from the eyelid } \\
\text { Group 3: Posterior lamellar } \\
\text { tarsal rotation }\end{array}$ \\
\hline Key eligibility criteria & $\begin{array}{l}\text { Inclusion criteria: } \\
\text { At least one eyelid with } \\
\text { previously unoperated upper } \\
\text { eyelid TT } \\
\text { Aged 18+years } \\
\text { Willingness to comply with } \\
\text { all study procedures and be } \\
\text { randomized to one of three } \\
\text { surgical procedures } \\
\text { No plans to move from the } \\
\text { region for the duration of the } \\
\text { study } \\
\text { Exclusion criteria: } \\
\text { Inability to provide } \\
\text { independent, informed } \\
\text { consent } \\
\text { Eyes with previously } \\
\text { unoperated TT are phthisical }\end{array}$ \\
\hline Study type & Randomised controlled trial \\
\hline Date of first enrollment & April 5, 2017 \\
\hline Target sample size & 6920 eyes \\
\hline Recruitment status & Completed \\
\hline Primary outcome(s) & Postoperative trichiasis \\
\hline Key secondary outcomes & $\begin{array}{l}\text { Eye contour abnormality and } \\
\text { granuloma }\end{array}$ \\
\hline
\end{tabular}


multiple graders assess the same set of images and then having the same grader re-grade the images a month later.

\section{ETHICS AND DISSEMINATION}

The National Research Ethics Review Committee of Ethiopia and Food Medicine Healthcare and Control Authority of Ethiopia, as well as the Institutional Review Boards at UNC and Johns Hopkins School of Medicine provided approval for the study, which is renewed annually. All protocol modifications are reported to the ethics boards prior to implementation. The current protocol version is 2.2. The trial is registered at ClinicalTrials.gov Identifier: NCT03100747. Further details are provided in table 3. On completion, trial results will be disseminated at local and international meetings and in peer-reviewed journals.

\section{Author affiliations}

${ }^{1}$ Orbis International Ethiopia, Addis Ababa, Ethiopia

${ }^{2}$ Department of Epidemiology, University of North Carolina at Chapel Hill, Chapel Hill, North Carolina, USA

${ }^{3}$ Department of Ophthalmology and Visual Sciences, University of Maryland School of Medicine, Baltimore, Maryland, USA

${ }^{4}$ Department of Ophthalmology, Johns Hopkins School of Medicine, Baltimore,

Maryland, USA

${ }^{5}$ Epidemiology Branch, National Institute of Environmental Health Sciences, Durham, North Carolina, USA

${ }^{6}$ Ophthalmology, University of North Carolina at Chapel Hill, Chapel Hill, NC, United States

Acknowledgements We would like to acknowledge the MTSS study staff for their dedication and their tireless effort put forth to provide high-quality, compassionate care to the thousands of trichiasis patients who received surgery. We are grateful for Orbis for coordinating the research with existing programs and also proving managerial and technical support.

Contributors All authors meet all four criteria for authorship. Category 1: EG, SLM, $B M$ and $A S$ designed the study. BB, AS, KMS, SLM, EG acquired data. BM, AK, AS, EG, SLM are responsible for data analysis and interpretation. Category 2: BB and KMS drafted the initial manuscript. All authors revised the manuscript for important intellectual content. Category 3: All authors provided final approval of the version to be published. Category 4: All authors agree to be accountable for all aspects of the work in ensuring that questions related to the accuracy or integrity of any part of the work are appropriately investigated and resolved.

Funding This work is supported by the National Eye Institute (301-496-5248) UG1EY025992. The study is funded under a cooperative agreement. Through this mechanism, the funder has access to data but does not influence decisions regarding data interpretation or publications. Study results will be published in a peer-reviewed journal and presented at both in-country and international meetings of trachoma experts.

Competing interests None declared.

Patient consent for publication Obtained.

Provenance and peer review Not commissioned; externally peer reviewed.

Open access This is an open access article distributed in accordance with the Creative Commons Attribution Non Commercial (CC BY-NC 4.0) license, which permits others to distribute, remix, adapt, build upon this work non-commercially, and license their derivative works on different terms, provided the original work is properly cited, appropriate credit is given, any changes made indicated, and the use is non-commercial. See: http://creativecommons.org/licenses/by-nc/4.0/.

\section{ORCID iD}

Emily W Gower http://orcid.org/0000-0003-1016-9910

\section{REFERENCES}

1 Geneva W. Progress report for the global elimination of blinding trachoma by the year 2020. Wkly Epidemiol Rec 2014;89:421-8.

2 World report on vision. World Health organization, 2019. Available: https://www.who.int/publications-detail/world-report-on-vision [Accessed 12 Nov 2019].

3 Who alliance for the global elimination of trachoma by 2020: progress report on elimination of trachoma, 2018. Wkly Epidemiol Rec 2019;94:317-28.

4 Merbs S, Resnikoff S, Kello S, et al. Trichiasis surgery for trachoma. 72. 2nd Ed, 2015.

5 West SK, West ES, Alemayehu W, et al. Single-Dose azithromycin prevents trichiasis recurrence following surgery: randomized trial in Ethiopia. Arch Ophthalmol 2006;124:309-14.

6 Gower EW, West SK, Harding JC, et al. Trachomatous trichiasis clamp vs standard bilamellar tarsal rotation instrumentation for trichiasis surgery: results of a randomized clinical trial. JAMA Ophthalmol 2013;131:294-301.

7 Burton MJ, Rajak SN, Ramadhani A, et al. Post-Operative recurrent trachomatous trichiasis is associated with increased conjunctival expression of S100A7 (psoriasin). PLoS Negl Trop Dis 2012;6:e1985.

8 Habtamu E, Rajak SN, Tadesse Z, et al. Epilation for minor trachomatous trichiasis: four-year results of a randomised controlled trial. PLoS Negl Trop Dis 2015;9:e0003558.

9 Habtamu E, Wondie T, Aweke S, et al. Posterior lamellar versus bilamellar tarsal rotation surgery for trachomatous trichiasis in Ethiopia: a randomised controlled trial. Lancet Glob Health 2016;4:e175-84.

10 Rajak SN, Habtamu E, Weiss HA, et al. Absorbable versus silk sutures for surgical treatment of trachomatous trichiasis in Ethiopia: a randomised controlled trial. PLoS Med 2011;8:e1001137.

11 Rajak SN, Habtamu E, Weiss HA, et al. Surgery versus epilation for the treatment of minor trichiasis in Ethiopia: a randomised controlled noninferiority trial. PLoS Med 2011;8:e1001136.

12 Khandekar R, Al-Hadrami K, Sarvanan N, et al. Recurrence of trachomatous trichiasis 17 years after bilamellar tarsal rotation procedure. Am J Ophthalmol 2006;141:1087-91.

13 Khandekar R, Thanh TT, Luong Q. The determinants of trichiasis recurrence differ at one and two years following lid surgery in Vietnam: a community-based intervention study. Oman J Ophthalmol 2009;2:119.

14 Rajak SN, Makalo P, Sillah A, et al. Trichiasis surgery in the Gambia: a 4-year prospective study. Invest Ophthalmol Vis Sci 2010;51:4996-5001.

15 Rajak SN, Habtamu E, Weiss HA, et al. The outcome of trachomatous trichiasis surgery in Ethiopia: risk factors for recurrence. PLoS Negl Trop Dis 2013;7:e2392.

16 Ramyil A, Bascaran C, Bunce C, et al. Outcome of trachoma lid surgeries in Jigawa state, Nigeria. Cogent Medicine 2016;3.

17 Habtamu E, Wondie T, Aweke S, et al. Oral doxycycline for the prevention of postoperative trachomatous trichiasis in Ethiopia: a randomised, double-blind, placebo-controlled trial. Lancet Glob Health 2018;6:e579-92.

18 Alemayehu W, Melese M, Bejiga A, et al. Surgery for trichiasis by ophthalmologists versus integrated eye care workers: a randomized trial. Ophthalmology 2004;111:578-84.

19 Gower EW, Merbs SL, Munoz BE, et al. Rates and risk factors for unfavorable outcomes 6 weeks after trichiasis surgery. Invest Ophthalmol Vis Sci 2011;52:2704-11.

20 ICTC. Global Scientific Meeting on Trachomatous Trichiasis. Int Coalit Trach Control, 20127. Available: https://www.who.int/ neglected_diseases/resources/Moshi_TTSWR_English_2013.4.19/ en/ [Accessed 6 Sep 2019].

21 Merbs SL, Kello AB, Gelema H, et al. The trachomatous trichiasis clamp: a surgical instrument designed to improve bilamellar tarsal rotation procedure outcomes. Arch Ophthalmol 2012;130:220-3.

22 Merbs SL, Oktavec KC, Munoz BE, et al. Lower postoperative scar height is associated with increased postoperative Trichiasis 1 year after bilamellar tarsal rotation surgery. Ophthalmic Epidemiol 2015;22:200-7.

23 Gower EW, West SK, Cassard SD, et al. Definitions and standardization of a new grading scheme for eyelid contour abnormalities after trichiasis surgery. PLoS Negl Trop Dis 2012;6:e1713.

24 Gower EW, Kello AB, Kollmann KM, Martin Kollmann KH. Training trichiasis surgeons: ensuring quality. Community Eye Health 2014;27:58.

25 Tadesse D, Montgomery I, Sankar G. HEAD START - an innovative training approach for life-long learning. Community Eye Health 2017;30:14. 
26 Dawson CR, Jones BR, Tarizzo ML. Guide to trachoma control in programmes for the prevention of blindness 1981 .
27 Rajak SN, Habtamu E, Weiss HA, et al. The clinical phenotype of trachomatous trichiasis in Ethiopia: not all trichiasis is due to entropion. Invest Ophthalmol Vis Sci 2011;52:7974. 